\title{
Structure Parameters and Displacement Equations of Goldberg Mechanisms With the Rank More Than sixStructure Parameters and Displacement Equations of Goldberg Mechanisms With the Rank More Than Six
}

\author{
Yitong Zhang \\ Yanshan University \\ Ling Lu ( $\nabla 1650680028 @ q q . c o m$ ) \\ Yanshan University \\ Wenlan Liu \\ Lanzhou University of Technology \\ Yanwen Li \\ Yanshan University \\ Bo Hu \\ Yanshan University \\ Dejun Mu \\ yanshan university \\ Wenjuan Lu \\ Yanshan University
}

Original Article

Keywords: Goldberg mechanism, Bennett mechanism, Mechanism with the rank more than six, Closedloop displacement equation

Posted Date: September 17th, 2020

DOI: https://doi.org/10.21203/rs.3.rs-72243/v1

License: (c) (1) This work is licensed under a Creative Commons Attribution 4.0 International License.

Read Full License 


\section{Title page}

\section{Structure parameters and displacement equations of Goldberg mechanisms with the rank more than six}

Yi-Tong Zhang, born in 1945, is currently a professor at School of Mechanical Engineering, Yanshan University, China. His research interest is the theory of mechanism structure.

E-mail: ytzhang@ysu.edu.cn

Ling Lu, born in 1964, is currently an associate professor at School of Mechanical Engineering, Yanshan University, China. She received her PhD degree from Yanshan University, China, in 2012. Her research interests include advanced manufacturing technology and robotics. E-mail: luling@ysu.edu.cn

Wen-Lan Liu, born in 1990, is currently a teacher at Lanzhou University of Technology, China. She received her PhD degree from Yanshan University, China, in 2018. Her research interests include theory and application of parallel mechanisms.

E-mail: wenlanl@163.com

Yan-Wen Li, born in 1966, is currently a professor at School of Mechanical Engineering, Yanshan University, China. She received her $\mathrm{PhD}$ degree from Yanshan University, China. Her research interests include technology and application of robots.

E-mail: ywl@ysu.edu.cn

Bo Hu, born in 1982, is currently a professor at School of Mechanical Engineering, Yanshan University, China. He received his PhD degree from Yanshan University, China, in 2010. His research interests include theory of mechanism structure and robotics.

E-mail: hubo@ysu.edu.cn

De-Jun Mu, born in 1967, is currently an associate professor at School of Mechanical Engineering, Yanshan University, China. She received her PhD degree from Yanshan University, China. Her research interest is the theory of mechanism structure.

E-mail: djmu@ysu.edu.cn

Wen-Juan Lu, born in 1983, is currently an associate professor at School of Mechanical Engineering, Yanshan University, China. She received her PhD degree from Yanshan University, China, in 2015. Her research interest is the theory of mechanism structure.

E-mail: luwenjuan@ysu.edu.cn

\section{Corresponding author: Ling Lu E-mail: luling@ysu.edu.cn}




\title{
Structure Parameters and Displacement Equations of Goldberg Mechanisms with the Rank More Than Six
}

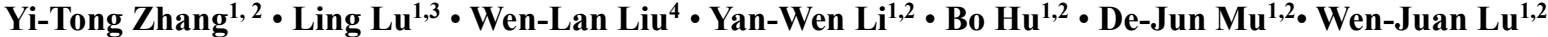

Received August 18, 2020; revised xx xx, 202x; accepted xx xx, 202x

(c) Chinese Mechanical Engineering Society and Springer-Verlag Berlin Heidelberg 2017

\begin{abstract}
An infinite number of $N$-bar Goldberg mechanisms with the rank more than six are proposed. A single closed-loop eight-bar mechanism is first developed by removing the common side links of adjacent loops from the five-loop Bennett mechanism in which all loop frames are collinear. An analysis of trajectory circles of the links shows that the mechanism developed maintains the original constraint conditions and kinematic characteristics, and that its degree of freedom (DOF) is also one. This novel single-loop mechanism is named the eight-bar Goldberg mechanism because of its similar constraint characteristics with the five-bar and six-bar Goldberg mechanisms. The eight displacement parameters of this mechanism are analyzed by using the interior angles of adjacent links, and the five cosine equations for the tangent product of the stagger angles between adjacent links are established in together with one angular displacement equation of odd links and one angular displacement equation of even links. This novel mechanism has one DOF, eight displacement parameters and seven independent displacement equations. The loop rank of the eightbar Goldberg mechanism is seven, which revises the existing mechanism theory of single closed-loop mechanisms with the rank no more than six. Similarly, a nine-bar Goldberg mechanism is constructed, and its nine displacement parameters are analyzed. This mechanism has eight independent displacement equations and the loop rank is eight, proving that the mechanisms with the rank more than six are not rare. Then, according to the characteristics of the independent displacement equations of the eight-bar and ninebar Goldberg mechanisms, the $N(N>7)$ displacement parameters of $N$-bar Goldberg mechanisms are analyzed, and the $(N-1)$

Ling Lu

luling@ysu.edu.cn

1 Parallel Robot and Mechatronic System Laboratory of Hebei Province, Yanshan University, Qinhuangdao 066004, China

2 Key Laboratory of Advanced Forging \& Stamping Technology and Science of Ministry of National Education, Yanshan University,
\end{abstract}

independent displacement equations are deduced with odd and even $N$ s. It is proved that the loop rank of $N$-bar Goldberg mechanisms is $(N-1)$, so there exist an infinite number of mechanisms with the rank more than six.

Keywords: Goldberg mechanism - Bennett mechanism • Mechanism with the rank more than six - Closed-loop displacement equation

\section{Introduction}

In 1903, Bennett invented a 4R spatial linkage mechanism [1-4], i.e., the Bennett mechanism (Figure 1), in which $a$ and $\alpha$ represent the length and stagger angle of side links $A B$ and $D C$, respectively, and $b$ and $\beta$ denote the length and stagger angle of link $B C$ and frame $A D$, respectively. In 1931, Myard proposed the plane-symmetric five-bar Myard mechanism by removing the common side link $A B$ from the two-loop Bennett mechanism (Figure 2) [5-6]. Goldberg invented the five-bar Goldberg mechanism in 1943 by using Myard's deletion method to delete the common side link of the mechanism after merging the loop I and II in Figure 3, similarly, the six-bar Goldberg mechanism was obtained [712]. These two Goldberg mechanisms are overconstrained, with the overconstraints being two and one, respectively. They have one DOF and the same constraint conditions and kinematic characteristics as their original multi-loop Bennett mechanisms. The loop ranks of the five-bar and sixbar mechanisms are four and five, respectively.

Qinhuangdao 066004, China

3 Heavy-duty Intelligent Manufacturing Equipment Innovation Center of Hebei Province, Yanshan University, Qinhuangdao 066004, China

4 College of Energy and Power Engineering, Lanzhou University of Technology, Lanzhou 730050, China 


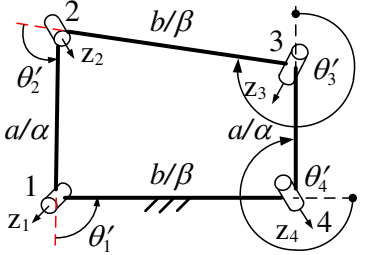

Figure 1 Bennett mechanism expressed by exterior angles

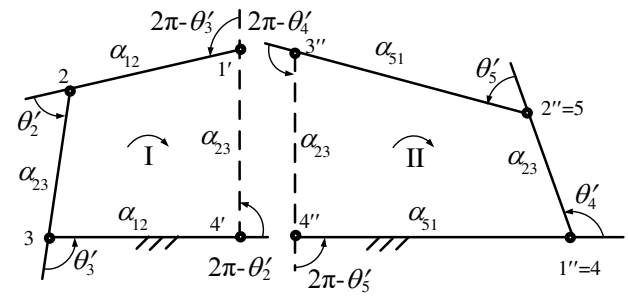

Figure 3 Five-bar Goldberg mechanism expressed by exterior angles

The loop rank is the most important parameter in the DOF analysis of a mechanism. In the 35 important formulas about DOFs from 1854 to 2003 summarized by Gogu [13] and other important formulas proposed after 2003 [14-25], the loop rank $(d)$ of a mechanism is a natural number no more than six. Without exception, those formulas about DOFs are all established under the condition of $d \leq 6$, so the conclusion that the loop rank of a mechanism is no more than six is generally accepted.

There are two reasons for the conclusion that $d \leq 6$ : (1) Twelve equations can be obtained in the rectangular coordinate system according to the 4-order H-D matrix of the closed loop of a mechanism, of which three are the independent displacement equations and nine are dependent rotation equations. Among the nine rotation equations, only three are independent, so there are at most six independent equations can be obtained from the 4-order H-D matrix, and the loop rank corresponding to the number of those independent equations is no more than six [26-27]. (2) At present, the screw theory is considered to be one of the most effective mathematical tools to analyze the DOF of mechanisms. The loop rank is defined by the rank of the kinematic screws or the number of the common constraint wrenches $[14,16,28]$, and both of them are less than or equal to six. Thus, the loop rank of a mechanism is also no more than six, i.e., $d \leq 6$.

In this year, based on the existing research of the five-bar and six-bar Goldberg mechanisms, we used Myard's deletion method to remove the common side links of adjacent loops from the five-loop, six-loop, ..., and nineloop Bennett mechanisms in which all loop frames are collinear, and the eight-bar, nine-bar, ..., and twelve-bar single-loop mechanisms with one DOF were developed. According to the DOF calculation formula ( $F=\sum_{i=1}^{N} f_{i}-d_{N}$ ) of a single-loop $N$-bar mechanism, the loop rank $\left(d_{N}\right)$ of the mechanism can be calculated by $d_{N}=\sum_{i=1}^{N} f_{i}-F=N-1$, where $f_{i}$ represents the number of single-DOF possessed by the $i$-th kinematic joint. Thus, if $N=8,9, \ldots, 12$, the loop ranks of the corresponding mechanisms are $7,8, \ldots, 11$, respectively.

No matter the rank of kinematic screws or the number of the common constraint wrenches is used to define the loop rank of a mechanism with the rank more than six, it will be less than or equal to six. Therefore, the method of defining the loop rank of mechanisms by the screw theory is no longer applicable after finding out the mechanisms with the rank more than six.

Moroskine defined in 1954 that the loop rank of a mechanism is equal to the number of its independent displacement equations. Whether this definition is applicable to the mechanisms with the rank more than six depends on whether the number of the independent displacement equations is equal to the loop rank of the mechanisms. To confirm this problem, the closed-loop displacement equations of the mechanisms with the rank more than six will be analyzed below.

\section{Formation and Characteristics of the Eight-, Nine-, ..., and $N$-bar Goldberg Mechanisms}

The five-loop Bennett mechanism with collinear loop frames is shown in Figure 4, in which there are 16 revolution joints including the composite joints $C, D, E$ and $F$. Let $L$ and $d_{j}$ represent the number of the closed loops and the rank of the $j$-th loop, respectively. Thus, the number of the DOF of the five-loop Bennett mechanism can be calculated by $F=\sum_{i=1}^{N} f_{i}-\sum_{j=1}^{L} d_{i}=16-3 \times 5=1$, where, $L=5$ and $d_{j}=3$. The centers of revolution joints in the mechanism are denoted by $A, B, C, D, E, F, G, H, O_{F}, O_{E}, O_{D}$, and $O_{C}$, respectively, and the length of the side links is represented by $a$. Taking points $O_{C}, O_{D}, O_{E}$, and $O_{F}$ as the centers, and length $a$ as the radius, the trajectory circles of points $C, D$, $E$, and $F$ are drawn, denoted by $S_{C}, S_{D}, S_{E}$, and $S_{F}$, respectively, as shown in Figure 4. A single-loop eight-bar mechanism is obtained by removing the common side links $9,10,11$, and 12 of adjacent loops from the five-loop Bennett mechanism, as shown in Figure 5. If side link 1 of the eight-bar mechanism is driven, the trajectories of points $C, D, E$, and $F$ are still the circles $S_{C}, S_{D}, S_{E}$, and $S_{F}$, respectively. That is, the single-loop eight-bar mechanism maintains the constraint conditions and kinematic characteristics of the five-loop Bennett mechanism, and it also has one DOF. 


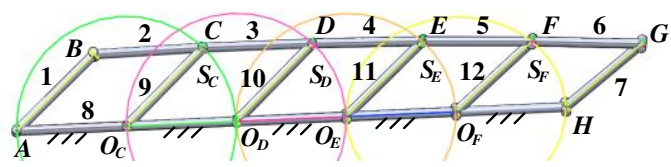

Figure 4 Five-loop Bennett mechanism

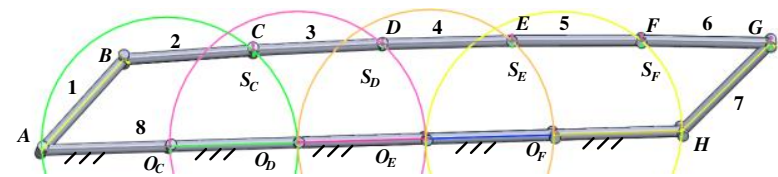

Figure 5 Eight-bar Goldberg mechanism

Like the five-bar and six-bar Goldberg mechanisms with collinear loop frames, the single-loop eight-bar mechanism also satisfies the constraint conditions of Bennett mechanism and has one DOF. In order to commemorate Goldberg's invention and maintain the consistency of the theoretical system of mechanisms, the novel single-loop eight-bar mechanism is named the eight-bar Goldberg mechanism.

Since the number of DOF of a mechanism is the difference between the total number of single-DOF possessed by the kinematic joints and the closed-loop rank of the mechanism, that of the eight-bar Goldberg mechanism can be expressed as $F=\sum_{i=1}^{N} f_{i}-d_{8}=1$, where $F, f_{i}$, and $d_{8}$ represent the number of DOF, number of single DOFs possessed by the $i$-th kinematic joint, and loop rank, respectively. Thus, $d_{8}$ can be derived as

$$
d_{8}=\sum_{i=1}^{N} f_{i}-F=8-1=7 .
$$

Eq. (1) shows that the eight-bar Goldberg mechanism is a single-loop mechanism with the rank more than six, which is an amazing result. In the theory of mechanisms, it is generally accepted that the rank of a single closed-loop is less than or equal to six. That is, the rank cannot be greater than six. No single closed-loop mechanism with the loop rank greater than six has been found before the proposal of the eight-bar Goldberg mechanism.

A six-loop Bennett mechanism is shown in Figure 6, in which all loop frames are collinear. Side links 10, 11, 12, 13, and 14 shared by adjacent loops of the six-loop Bennett mechanism are removed, resulting in a single-loop nine-bar mechanism, as shown in Figure 7. When side link 1 is driven, points $C, D, E, F$, and $G$ in the nine-bar mechanism still move on the trajectory circles $S_{C}, S_{D}, S_{E}, S_{F}$, and $S_{G}$, respectively. These movements are the same as those in the six-loop Bennett mechanism. Therefore, the single-loop nine-bar mechanism maintains the constraint conditions and kinematic characteristics of the six-loop Bennett mechanism, and also has one DOF. Similarly, it is named the nine-bar
Goldberg mechanism, and its loop rank can be calculated as $d_{9}=\sum_{i=1}^{N} f_{i}-F=9-1=8>6$, showing that the mechanisms with the rank more than six are not individual.

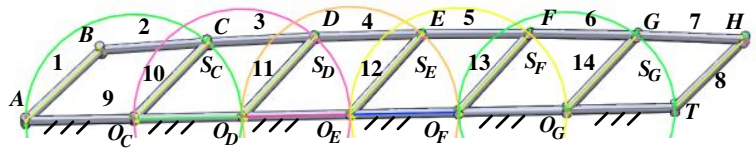

Figure 6 Six-loop Bennett mechanism

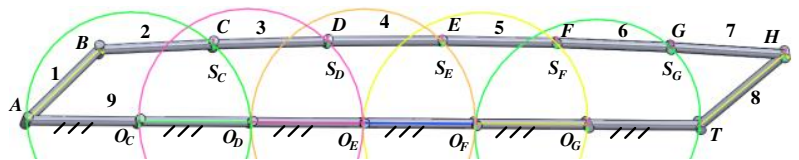

Figure 7 Nine-bar Goldberg mechanism

Similarly, the Bennett mechanism is successively added to the six-loop mechanism, and the side links shared by adjacent loops are removed. A series of single-loop mechanisms with ten, eleven, ..., and $N$ links can be obtained, which also have one DOF and maintain the constraint conditions of Bennett mechanism. Thus, these mechanisms are named the ten-bar, eleven-bar, ..., and $\mathrm{N}$ bar Goldberg mechanisms, respectively, which form an infinite number of mechanisms with the rank more than six.

The important theoretical significance of the $N$-bar Goldberg mechanisms is that they are the only type of mechanisms with the rank more than six discovered for the first time. The theory that the rank of the closed-loop mechanisms is less than or equal to six is broken through. Those mechanisms show that the rank of mechanisms can not only be greater than six, but also be infinite.

\section{Traditional and New Methods for Express- ing Displacement Equations of Bennett Mechanism}

The displacement equations of Bennett and Goldberg mechanisms are usually expressed by exterior angles of adjacent links [29-36]. To reflect more intuitively and clearly the geometric relationship between the links in the analysis of multiple loops, interior angles of adjacent links are adopted in this paper. For the convenience of reading, the traditional displacement equations of Bennett mechanism and the five-bar Goldberg mechanism are introduced first, as well as the difference between them and that expressed by interior angles. 


\subsection{Traditional Method for Expressing Structure Parameters and Displacement Equations of Bennett Mechanism}

\subsubsection{Structure Parameters of Bennett Mechanism Expressed by the Traditional Method}

The geometric constraint conditions of Bennett mechanism (Figure 1) given by Bennett in 1914 [4] are as follow: (1) the links on the opposite sides are equal in length and stagger angles, and the rotation directions of the two stagger angles are the same, (2) the length ratio of two adjacent links is equal to the sine ratio of the corresponding two stagger angles. The lengths of links 1, 2, 3, and 4 of the Bennett mechanism shown in Figure 1 are denoted by $a_{1}, b_{2}, a_{3}$, and $b_{4}$, respectively. The stagger angles of links 1 and 4,1 and 2, 2 and 3, and 3 and 4 are represented by $\alpha_{1}, \beta_{2}, \alpha_{3}$, and $\beta_{4}$, respectively. Then the geometric constraint conditions can be expressed as

$$
\begin{gathered}
a_{1}=a_{3}=a, b_{2}=b_{4}=b, \alpha_{1}=\alpha_{3}=\alpha, \text { and } \beta_{2}=\beta_{4}=\beta, \\
\frac{a}{b}=\frac{\sin \alpha}{\sin \beta} .
\end{gathered}
$$

Eq. (3) is the proportion equation of adjacent links, where the range of the stagger angles $\alpha$ and $\beta$ is $\left(0^{\circ}, 180\right)$. In addition, the values of $\alpha$ and $\beta$ cannot be selected in the same quadrant, which is called the different quadrant rule of stagger angles in this paper.

\subsubsection{Displacement Equations of Bennett Mechanism Expressed by the Traditional Method}

Since the Bennett mechanism shown in Figure 1 is designed according to the different quadrant rule of stagger angles, the axes of $z_{1}$ and $z_{4}$ are in the opposite direction, so are axes $z_{2}$ and $z_{3}$. It is assumed that the rotation angle of two adjacent links takes a positive value in the anti-clockwise direction and a negative value in the clockwise direction from the view of the positive direction of the $z$ axis. Thus, when the exterior angle of axis $z_{1}$ is less than $180^{\circ}$, that of axis $z_{3}$ is greater than $180^{\circ}$. The relationship between the exterior angles of axes $z_{2}$ and $z_{4}$ is similar to that of axes $z_{1}$ and $z_{3}$. Under this assumption, the following two linear equations and a nonlinear trigonometric equation were given in [4]:

$$
\begin{gathered}
\theta_{1}^{\prime}+\theta_{3}^{\prime}=2 \pi, \\
\theta_{2}^{\prime}+\theta_{4}^{\prime}=2 \pi, \\
\tan \frac{\theta_{1}^{\prime}}{2} \tan \frac{\theta_{2}^{\prime}}{2}=\frac{\sin \left(\frac{\beta+\alpha}{2}\right)}{\sin \left(\frac{\beta-\alpha}{2}\right)}=k,
\end{gathered}
$$

where $\theta_{1}^{\prime}, \theta_{2}^{\prime}, \theta_{3}^{\prime}$, and $\theta_{4}^{\prime}$ denote the exterior angles of axes $z_{1}, z_{2}, z_{3}$, and $z_{4}$, respectively. Eq. (6) is the sine equation for the tangent product of the exterior angles of adjacent links, where $k$ is the sine constant of the tangent product of exterior angles.

\subsection{Displacement Equations Expressed by Interior Angles of Bennett Mechanism}

\subsubsection{Structure Parameters of Bennett Mechanism with} Acute Stagger Angles

To express more intuitively and simply the geometric relationship between the angular displacements of the mechanisms with the rank more than six, the displacement equations expressed by interior angles of Bennett mechanism are proposed in this paper.

The stagger angle of two adjacent links of Bennett mechanism is defined as an acute angle, and its value range is $\left(-90^{\circ}, 90^{\circ}\right)$. The geometric conditions of the mechanism are as follows: (1) the links on the opposite sides are equal in length and stagger angles, (2) the length ratio of two adjacent links is equal to the negative sine ratio of the two corresponding stagger angles, (3) the values of stagger angles of adjacent links cannot be selected in the same quadrant of the range. The geometric conditions of Bennett mechanism can be reformulated into

$$
\begin{gathered}
a_{1}=a_{3}=a, \alpha_{1}=\alpha_{3}=\alpha, b_{2}=b_{4}=b, \beta_{2}=\beta_{4}=\beta, \\
\frac{a}{b}=-\frac{\sin \alpha}{\sin \beta}, \\
\boldsymbol{\tau}_{a}=-\tau_{b},\left(\left|\boldsymbol{\tau}_{a}\right|=\left|\boldsymbol{\tau}_{b}\right|=1\right),
\end{gathered}
$$

where $\boldsymbol{\tau}_{a}$ and $\boldsymbol{\tau}_{b}$ denote the unit vectors of the stagger angles of the side links and the link connected to the side links relative to the frame, respectively. $\tau_{a}$ and $\tau_{b}$ take a positive value in the anti-clockwise direction and a negative value in the clockwise direction. Eq. (9) emphasizes that the directions of the stagger angles of two adjacent links are opposite.

There are two types of Bennett mechanism: right-handed (i.e., in the same direction as the right-hand thread) and lefthanded. For the former, the stagger angles $\alpha$ and $\beta$ satisfy $-90^{\circ}<\alpha<0^{\circ}$ and $0^{\circ}<\beta<90^{\circ}$, for example, the mechanism shown in Figure 8, and for the latter, $0^{\circ}<\alpha<90^{\circ}$ and $-90^{\circ}<\beta<0^{\circ}$. 


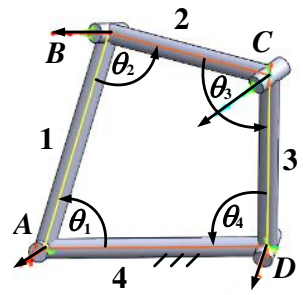

Figure 8 Bennett mechanism expressed by interior angles

\subsubsection{Displacement Equations of Bennett Mechanism Expressed by Interior Angles}

Let $\theta_{i}(i=1,2,3,4)$ be the angular displacement of the $i$-th link relative to its previous adjacent link, which is also the interior angle of two adjacent links of Bennett mechanism. Thus, the range of $\theta_{i}$ is $-180^{\circ} \leq \theta_{l} \leq 180^{\circ}$.

The angles between adjacent links are expressed by the interior angles, then the closed-loop displacement equations of Bennett mechanism can be formulated as

$$
\begin{gathered}
\theta_{1}=\theta_{3}\left(\text { or } \theta_{1}-\theta_{3}=0\right), \\
\theta_{2}=\theta_{4}\left(\text { or } \theta_{2}-\theta_{4}=0\right), \\
\tan \frac{\theta_{1}}{2} \tan \frac{\theta_{2}}{2}=\frac{\cos \left(\frac{\beta-\alpha}{2}\right)}{\cos \left(\frac{\beta+\alpha}{2}\right)}=k .
\end{gathered}
$$

Eq. (12) is the cosine equation for the tangent product of interior angles of adjacent links, where $k$ is the cosine constant of the tangent product of interior angles.

\subsection{Application and Shortage of Traditional Displacement Equations of Bennett Mechanism in Multi-loop Mechanisms}

\subsubsection{Traditional Displacement Equations of the Five-bar} Goldberg Mechanism

The five-bar Goldberg mechanism shown in Figure 3 is composed of two traditional Bennett mechanisms by the authors of [8] in 1979, where the angular displacements of links are expressed by exterior angles. The traditional displacement equations of the five-bar Goldberg mechanism are given simply below. The readers can refer to [8] for the detailed analysis process.

One linear displacement equation can be obtained from $\left(2 \pi-\theta_{2}^{\prime}\right)+\left(2 \pi-\theta_{5}^{\prime}\right)=\pi$ that

$$
\theta_{2}^{\prime}+\theta_{5}^{\prime}=3 \pi
$$

Another linear displacement equation can be derived from $\left(2 \pi-\theta_{3}^{\prime}\right)+\left(2 \pi-\theta_{4}^{\prime}\right)=\pi+\theta_{1}^{\prime}$ that

$$
\theta_{1}^{\prime}+\theta_{3}^{\prime}+\theta_{4}^{\prime}=3 \pi
$$

For the five-bar Goldberg mechanism, the two sine equations for the tangent products of exterior angles of adjacent links can be expressed as

$$
\begin{aligned}
& \tan \frac{\theta_{2}^{\prime}}{2} \tan \frac{\theta_{3}^{\prime}}{2}=\frac{\sin \left(\frac{\alpha_{12}+\alpha_{23}}{2}\right)}{\sin \left(\frac{\alpha_{12}-\alpha_{23}}{2}\right)}, \\
& \tan \frac{\theta_{4}^{\prime}}{2} \tan \frac{\theta_{5}^{\prime}}{2}=\frac{\sin \left(\frac{\alpha_{51}+\alpha_{23}}{2}\right)}{\sin \left(\frac{\alpha_{51}-\alpha_{23}}{2}\right)} .
\end{aligned}
$$

\subsubsection{Displacement Equations of the Five-bar Goldberg} Mechanism Expressed by Interior Angles

According to the idea of Section 3.2, the angle displacements of links in Figure 3 are denoted by interior angles instead of exterior angles, as shown in Figure 9. It can be seen that the relationship between the angular displacements in Figure 9 is simpler and clearer than that in Figure 3.

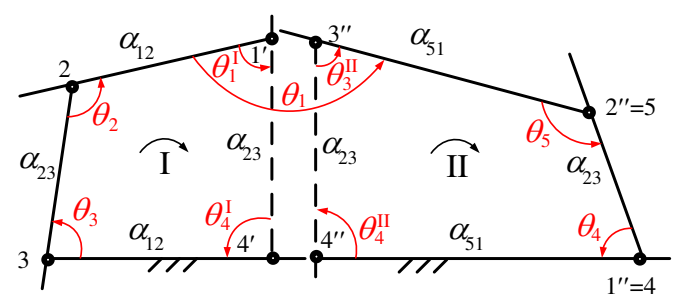

Figure 9 Five-bar Goldberg mechanism expressed by interior angles

Since $\theta_{4}^{\mathrm{I}}=\theta_{2}$ and $\theta_{4}^{\mathrm{II}}=\theta_{5}$, the follow linear equation can be obtained:

$$
\theta_{2}+\theta_{5}=\pi
$$

Taking $\theta_{1}^{\mathrm{I}}=\theta_{3} \quad$ and $\quad \theta_{3}^{\mathrm{II}}=\theta_{4} \quad$ in mind yields $\theta_{1}=\theta_{1}^{\mathrm{I}}+\theta_{3}^{\mathrm{II}}=\theta_{3}+\theta_{4}$, so another linear equation can be derived as:

$$
\theta_{1}-\theta_{3}-\theta_{4}=0
$$

The cosine equations for the tangent products of interior angles of adjacent links can be deduced from Eq. (12) as follows: 


$$
\begin{aligned}
& \tan \frac{\theta_{2}}{2} \tan \frac{\theta_{3}}{2}=\frac{\cos \left(\frac{\alpha_{12}-\alpha_{23}}{2}\right)}{\cos \left(\frac{\alpha_{12}+\alpha_{23}}{2}\right)}, \\
& \tan \frac{\theta_{4}}{2} \tan \frac{\theta_{5}}{2}=\frac{\cos \left(\frac{\alpha_{51}-\alpha_{23}}{2}\right)}{\cos \left(\frac{\alpha_{51}+\alpha_{23}}{2}\right)} .
\end{aligned}
$$

If link 3 of the five-bar Goldberg mechanism in Figure 9 is selected as the driving link, i.e., the value of $\theta_{3}$ is known, the values of $\theta_{1}, \theta_{2}, \theta_{4}$, and $\theta_{5}$ can be solved from the four independent Eqs. (17) (20).

Comparing the traditional displacement Eqs. (13) (16) and the displacement Eqs. (17) (20) expressed by interior angles of the five-bar Goldberg mechanism, the geometric relationship of the latter is very simple and clear, which brings great convenience for the establishment of displacement equations of multi-bar Goldberg mechanisms. Therefore, the displacement equations of the mechanisms with the rank more than six introduced later in this paper are all expressed by interior angles.

\section{Structure Parameters and Displacement Equations with Interior Angles of the Eight- bar Goldberg Mechanism}

\subsection{Structure Parameters of the Eight-bar Goldberg Mechanism}

An eight-bar Goldberg mechanism is shown in Figure 10. The length and stagger angle of side links 1 and 7 are denoted by $a$ and $\alpha$, respectively. The value range of $\alpha$ is $\left(-90^{\circ}, 90^{\circ}\right)$, taking a positive value in the anti-clockwise direction and a negative value in the converse direction. The length and stagger angle of the $j$-th $(j=1,2,3,4,5)$ link connected to side link 1 are denoted by $b_{j}$ and $\beta_{j}$, respectively. The value range and sign rule of $\beta_{j}$ are the same as those of $\alpha$. The total length and stagger angle of the frame are represented by $d_{r}$ and $\beta_{r}$, respectively. Like the Bennett mechanism, there are also two types of the eight-bar Goldberg mechanism: left-handed and right-handed. The right-handed mechanism is under the conditions of $-90^{\circ}<\alpha<0^{\circ}$ and $0^{\circ}<\beta_{j}<90^{\circ}$, as shown in Figures 5 and 10 . Otherwise, the mechanism is left-handed with $0^{\circ}<\alpha<90^{\circ}$ and $-90^{\circ}<\beta_{j}<0^{\circ}$.

The structure parameters of the eight-bar Goldberg mechanism can be expressed as

$$
\begin{gathered}
a_{1}=a_{7}=a, \quad \alpha_{1}=\alpha_{7}=\alpha, \\
d_{r}=\sum_{j=1}^{5} b_{j}, \text { and } \beta_{r}=\sum_{j=1}^{5} \beta_{j},
\end{gathered}
$$

$$
\begin{gathered}
\frac{\sin \alpha}{a}=-\frac{\sin \beta_{j}}{b_{j}} \quad(j=1,2,3,4,5), \\
\boldsymbol{\tau}_{a}=-\boldsymbol{\tau}_{b j},\left(\left|\boldsymbol{\tau}_{a}\right|=\left|\boldsymbol{\tau}_{b j}\right|=1\right)(j=1,2,3,4,5),
\end{gathered}
$$

where $\tau_{a}$ and $\tau_{b j}$ are the unit vectors of the stagger angles of the side links and the $j$-th link connected to side link 1 relative to the frame, respectively. They take a positive value in the anti-clockwise direction and a negative value in the clockwise direction. Eq. (23) emphasizes that the direction of the stagger angle between the $j$-th link and frame is opposite to that of the stagger angle between the side links and frame.

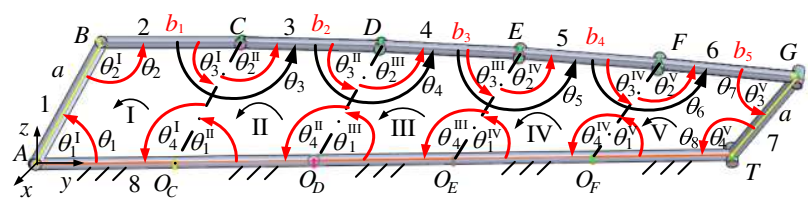

Figure 10 Displacement parameters of right-handed eight-bar Goldberg mechanism

\subsection{Displacement Equations of the Eight-bar Goldberg Mechanism Expressed by Interior Angles}

For the purpose of analysis, five virtual loops (numbered $\mathrm{I} \sim \mathrm{V})$ are constructed in the eight-bar Goldberg mechanism, as shown in Figure 10. Taking point $C$ as the object of analysis, there are $\theta_{3}=\theta_{3}^{\mathrm{I}}+\theta_{2}^{\mathrm{II}}=\theta_{1}+\theta_{4}^{\mathrm{II}}$ and $\theta_{4}^{\mathrm{II}}=\pi-\theta_{1}^{\mathrm{III}}=\pi-\left(\theta_{5}-\theta_{2}^{\mathrm{IV}}\right)=\pi-\theta_{5}+\left(\pi-\theta_{1}^{\mathrm{V}}\right)=2 \pi-\theta_{5}$ $-\theta_{7}$, i.e., $\theta_{3}=\theta_{1}+2 \pi-\theta_{5}-\theta_{7}$. Thus, the equation for the angular displacements of odd links can be derived as $\theta_{3}+\theta_{5}+\theta_{7}-\theta_{1}=2 \pi$. Taking point $D$ as the object of analysis, there exists $\theta_{4}=\theta_{3}^{\mathrm{II}}+\theta_{2}^{\mathrm{III}}=\pi-\theta_{2}+\left(\pi-\theta_{1}^{\mathrm{IV}}\right)$ $=\pi-\theta_{2}+\pi-\left(\theta_{6}-\theta_{2}^{\mathrm{V}}\right)=2 \pi-\theta_{2}-\theta_{6}+\theta_{8}$, so the equation for the angular displacements of even links can be obtained as $\theta_{2}+\theta_{4}+\theta_{6}-\theta_{8}=2 \pi$.

From virtual loops $\mathrm{I} \sim \mathrm{V}$ of the eight-bar Goldberg mechanism, the following five equations can be obtained:

$$
\begin{aligned}
& \tan \frac{\theta_{1}^{\mathrm{I}}}{2} \tan \frac{\theta_{2}^{\mathrm{I}}}{2}=\frac{\cos \left[\left(\beta_{1}-\alpha\right) / 2\right]}{\cos \left[\left(\beta_{1}+\alpha\right) / 2\right]}=k_{1}, \\
& \tan \frac{\theta_{1}^{\mathrm{II}}}{2} \tan \frac{\theta_{2}^{\mathrm{II}}}{2}=\frac{\cos \left[\left(\beta_{2}-\alpha\right) / 2\right]}{\cos \left[\left(\beta_{2}+\alpha\right) / 2\right]}=k_{2}, \\
& \tan \frac{\theta_{1}^{\mathrm{II}}}{2} \tan \frac{\theta_{2}^{\mathrm{III}}}{2}=\frac{\cos \left[\left(\beta_{3}-\alpha\right) / 2\right]}{\cos \left[\left(\beta_{3}+\alpha\right) / 2\right]}=k_{3}, \\
& \tan \frac{\theta_{1}^{\mathrm{IV}}}{2} \tan \frac{\theta_{2}^{\mathrm{IV}}}{2}=\frac{\cos \left[\left(\beta_{4}-\alpha\right) / 2\right]}{\cos \left[\left(\beta_{4}+\alpha\right) / 2\right]}=k_{4},
\end{aligned}
$$




$$
\tan \frac{\theta_{1}^{\mathrm{V}}}{2} \tan \frac{\theta_{2}^{\mathrm{V}}}{2}=\frac{\cos \left[\left(\beta_{5}-\alpha\right) / 2\right]}{\cos \left[\left(\beta_{5}+\alpha\right) / 2\right]}=k_{5},
$$

where $k_{1}, k_{2}, k_{3}, k_{4}$ and $k_{5}$ are constants.

Rearranging the above equations yields

$$
\begin{gathered}
\theta_{3}+\theta_{5}+\theta_{7}-\theta_{1}=2 \pi, \\
\theta_{2}+\theta_{4}+\theta_{6}-\theta_{8}=2 \pi, \\
\tan \frac{\theta_{1}}{2} \tan \frac{\theta_{2}}{2}=\frac{\cos \left[\left(\beta_{1}-\alpha\right) / 2\right]}{\cos \left[\left(\beta_{1}+\alpha\right) / 2\right]}=k_{1}, \\
\tan \frac{\pi-\theta_{2}}{2} \tan \frac{\theta_{3}-\theta_{1}}{2}=\frac{\cos \left[\left(\beta_{2}-\alpha\right) / 2\right]}{\cos \left[\left(\beta_{2}+\alpha\right) / 2\right]}=k_{2}, \\
\tan \frac{\theta_{1}-\theta_{3}}{2} \tan \frac{\theta_{4}+\theta_{2}-\pi}{2}=\frac{\cos \left[\left(\beta_{3}-\alpha\right) / 2\right]}{\cos \left[\left(\beta_{3}+\alpha\right) / 2\right]}=k_{3}, \\
\tan \frac{\theta_{6}-\theta_{8}}{2} \tan \frac{\pi-\theta_{7}}{2}=\frac{\cos \left[\left(\beta_{4}-\alpha\right) / 2\right]}{\cos \left[\left(\beta_{4}+\alpha\right) / 2\right]}=k_{4}, \\
\tan \frac{\theta_{7}}{2} \tan \frac{\theta_{8}}{2}=\frac{\cos \left[\left(\beta_{5}-\alpha\right) / 2\right]}{\cos \left[\left(\beta_{5}+\alpha\right) / 2\right]}=k_{5} .
\end{gathered}
$$

Eqs. (29) (35) are seven independent displacement equations expressed by eight angular displacement parameters (i.e., $\theta_{1}, \theta_{2}, \theta_{3}, \theta_{4}, \theta_{5}, \theta_{6}, \theta_{7}$, and $\theta_{8}$ ). Taking one of the eight displacement parameters as the input, the rest can be solved.

Multiplying Eqs. (31), (32), (33), (34), and (35) gives

$$
\tan \frac{\theta_{1}}{2} \tan \frac{\theta_{4}+\theta_{2}-\pi}{2} \tan \frac{\theta_{6}-\theta_{8}}{2} \tan \frac{\theta_{8}}{2}=k_{1} k_{2} k_{3} k_{4} k_{5}
$$

It can be deduced from Eq. (30) that

$$
\theta_{2}+\theta_{4}-\pi=\pi-\left(\theta_{6}-\theta_{8}\right)
$$

Substituting Eq. (37) into Eq. (36) yields

$$
\tan \frac{\theta_{1}}{2} \tan \frac{\theta_{8}}{2}=k_{1} k_{2} k_{3} k_{4} k_{5},
$$

which is just the input-output displacement equation expressed by the interior angles of side links 1 and 7 of the eight-bar Goldberg mechanism.

Since the number of independent displacement equations of a single closed-loop mechanism is equal to its loop rank [26-27,37-38], the loop rank of the eight-bar Goldberg mechanism is seven, i.e., $d_{8}=7>6$. This result sufficiently proves the existence of mechanisms with the rank more than six in theory. Alternatively, according to the trajectory circles of the links, the eight-bar Goldberg mechanism and the five-loop Bennett mechanism have the same motion; therefore, it can be derived from Eq. (1) that $d_{8}=7$. The results obtained by the two methods are consistent, so their correctness has been verified mutually. The eight-bar Goldberg mechanism with the loop rank being seven revises the traditional theory that the rank of a mechanism is no more than six.

\section{Structure Parameters and Displacement Equations of the Nine-bar Goldberg Mechanism}

\subsection{Structure Parameters of the Nine-bar Goldberg Mechanism}

The nine-bar Goldberg mechanism is shown in Figure 11, in which the symbols have the same meaning as those in the eight-bar Goldberg mechanism. Compared with the eightbar Goldberg mechanism, this mechanism has one more virtual loop and link. The structure parameter equations of the nine-bar Goldberg mechanism can be expressed as

$$
\begin{gathered}
a_{1}=a_{8}=a, \quad \alpha_{1}=\alpha_{8}=\alpha, \\
d_{r}=\sum_{j=1}^{6} b_{j}, \quad \beta_{r}=\sum_{j=1}^{6} \beta_{j}, \\
\frac{\sin \alpha}{a}=-\frac{\sin \beta_{j}}{b_{j}}(j=1,2,3,4,5,6), \\
\boldsymbol{\tau}_{a}=-\boldsymbol{\tau}_{b j},\left(\left|\boldsymbol{\tau}_{a}\right|=\left|\boldsymbol{\tau}_{b j}\right|=1\right)(j=1,2,3,4,5,6) .
\end{gathered}
$$

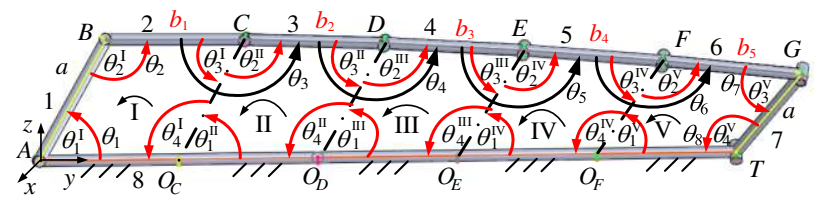

Figure 11 Displacement parameters of right-handed nine-bar Goldberg mechanism

\subsection{Displacement Equations of the Nine-bar Goldberg Mechanism}

Taking point $C$ of the nine-bar Goldberg mechanism as the object of analysis, $\theta_{3}=\theta_{3}^{\mathrm{I}}+\theta_{2}^{\mathrm{II}}=\theta_{1}+\theta_{4}^{\mathrm{II}}$ and $\theta_{4}^{\mathrm{II}}=\pi-$ $\theta_{1}^{\mathrm{III}}=\pi-\left(\theta_{5}-\theta_{2}^{\mathrm{IV}}\right)=\pi-\theta_{5}+\left(\pi-\theta_{1}^{\mathrm{V}}\right)=\pi-\theta_{5}+\pi-\left(\theta_{7}-\theta_{9}\right)$ $=2 \pi-\theta_{5}-\theta_{7}+\theta_{9}$. It can be obtained from these two equations that $\theta_{3}=\theta_{1}+2 \pi-\theta_{5}-\theta_{7}+\theta_{9}$. Therefore, the equation for the angular displacements of the odd links of the nine-bar Goldberg mechanism can be derived as $\theta_{3}+\theta_{5}+\theta_{7}-\theta_{9}-\theta_{1}=2 \pi$. Taking point $D$ as the object of analysis, $\quad \theta_{4}=\theta_{3}^{\mathrm{II}}+\theta_{2}^{\mathrm{III}}=\pi-\theta_{2}+\left(\pi-\theta_{1}^{\mathrm{IV}}\right)=\pi-\theta_{2}+\pi-$ 
$\left(\theta_{6}-\theta_{2}^{V}\right)=2 \pi-\theta_{2}-\theta_{6}+\left(\pi-\theta_{8}\right)$, from which the equation for the angular displacements of the even links can be obtained: $\theta_{2}+\theta_{4}+\theta_{6}+\theta_{8}=3 \pi$.

From the six virtual loops (numbered I VI) of the ninebar Goldberg mechanism, we can get

$$
\begin{aligned}
& \tan \frac{\theta_{1}^{\mathrm{I}}}{2} \tan \frac{\theta_{2}^{\mathrm{I}}}{2}=\frac{\cos \left[\left(\beta_{1}-\alpha\right) / 2\right]}{\cos \left[\left(\beta_{1}+\alpha\right) / 2\right]}=k_{1}, \\
& \tan \frac{\theta_{1}^{\mathrm{II}}}{2} \tan \frac{\theta_{2}^{\mathrm{II}}}{2}=\frac{\cos \left[\left(\beta_{2}-\alpha\right) / 2\right]}{\cos \left[\left(\beta_{2}+\alpha\right) / 2\right]}=k_{2}, \\
& \tan \frac{\theta_{1}^{\mathrm{II}}}{2} \tan \frac{\theta_{2}^{\mathrm{III}}}{2}=\frac{\cos \left[\left(\beta_{3}-\alpha\right) / 2\right]}{\cos \left[\left(\beta_{3}+\alpha\right) / 2\right]}=k_{3}, \\
& \tan \frac{\theta_{1}^{\mathrm{IV}}}{2} \tan \frac{\theta_{2}^{\mathrm{IV}}}{2}=\frac{\cos \left[\left(\beta_{4}-\alpha\right) / 2\right]}{\cos \left[\left(\beta_{4}+\alpha\right) / 2\right]}=k_{4}, \\
& \tan \frac{\theta_{1}^{\mathrm{V}}}{2} \tan \frac{\theta_{2}^{\mathrm{V}}}{2}=\frac{\cos \left[\left(\beta_{5}-\alpha\right) / 2\right]}{\cos \left[\left(\beta_{5}+\alpha\right) / 2\right]}=k_{5}, \\
& \tan \frac{\theta_{1}^{\mathrm{VI}}}{2} \tan \frac{\theta_{2}^{\mathrm{VI}}}{2}=\frac{\cos \left[\left(\beta_{6}-\alpha\right) / 2\right]}{\cos \left[\left(\beta_{6}+\alpha\right) / 2\right]}=k_{6} .
\end{aligned}
$$

Rearranging the above equations yields

$$
\begin{gathered}
\theta_{3}+\theta_{5}+\theta_{7}-\theta_{9}-\theta_{1}=2 \pi, \\
\theta_{2}+\theta_{4}+\theta_{6}+\theta_{8}=3 \pi, \\
\tan \frac{\theta_{1}}{2} \tan \frac{\theta_{2}}{2}=\frac{\cos \left[\left(\beta_{1}-\alpha\right) / 2\right]}{\cos \left[\left(\beta_{1}+\alpha\right) / 2\right]}=k_{1}, \\
\tan \frac{\pi-\theta_{2}}{2} \tan \frac{\theta_{3}-\theta_{1}}{2}=\frac{\cos \left[\left(\beta_{2}-\alpha\right) / 2\right]}{\cos \left[\left(\beta_{2}+\alpha\right) / 2\right]}=k_{2}, \\
\tan \frac{\pi-\left(\theta_{3}-\theta_{1}\right)}{2} \tan \frac{\theta_{4}+\theta_{2}-\pi}{2}=\frac{\cos \left[\left(\beta_{3}-\alpha\right) / 2\right]}{\cos \left[\left(\beta_{3}+\alpha\right) / 2\right]}=k_{3}, \\
\tan \frac{\theta_{7}-\pi}{2} \tan \frac{\pi-\left(\theta_{7}-\theta_{9}\right)}{2}=\frac{\cos \left[\left(\beta_{4}-\alpha\right) / 2\right]}{\cos \left[\left(\beta_{4}+\alpha\right) / 2\right]}=k_{4}, \\
\tan \frac{\pi-\theta_{8}}{2}=\frac{\cos \left[\left(\beta_{5}-\alpha\right) / 2\right]}{\cos \left[\left(\beta_{5}+\alpha\right) / 2\right]}=k_{5}, \\
\tan \frac{\theta_{8}}{2} \tan \frac{\theta_{9}}{2}=\frac{\cos \left[\left(\beta_{6}-\alpha\right) / 2\right]}{\cos \left[\left(\beta_{6}+\alpha\right) / 2\right]}=k_{6} .
\end{gathered}
$$

Eqs. (48) (55) are eight independent displacement equations expressed by nine angular displacement parameters (i.e., $\theta_{1}, \theta_{2}, \theta_{3}, \theta_{4}, \theta_{5}, \theta_{6}, \theta_{1}, \theta_{8}$, and $\theta_{9}$ ). Taking one of the nine displacement parameters as the input, the rest can be solved.

Multiplying Eqs. (50) (55) and using the equations $\theta_{2}+\theta_{4}+\theta_{6}+\theta_{8}=3 \pi$ and $\tan \frac{\theta_{4}+\theta_{2}-\pi}{2} \tan \frac{\theta_{6}+\theta_{8}-\pi}{2}$ $=\tan \frac{\theta_{4}+\theta_{2}-\pi}{2} \tan \frac{2 \pi-\left(\theta_{4}+\theta_{2}\right)}{2}=1$ lead to

$$
\begin{aligned}
\tan \frac{\theta_{1}}{2} \tan \frac{\theta_{9}}{2} & =\frac{\cos \left[\left(\beta_{1}-\alpha\right) / 2\right]}{\cos \left[\left(\beta_{1}+\alpha\right) / 2\right]} \cdots \frac{\cos \left[\left(\beta_{6}-\alpha\right) / 2\right]}{\cos \left[\left(\beta_{6}+\alpha\right) / 2\right]} \\
& =k_{1} k_{2} \cdots k_{6}
\end{aligned}
$$

which is just the input-output displacement equation expressed by the interior angles of side links 1 and 8 of the nine-bar Goldberg mechanism.

\section{Structure Parameters and Displacement Equations of $N$-bar Goldberg Mechanisms}

In view of the similarities and differences between the displacement equations of the eight-bar and nine-bar Goldberg mechanisms, the $N$-bar Goldberg mechanisms are divided into two categories: one with odd links and the other with even links. Then, the general displacement equations of $N$-bar Goldberg mechanisms are derived via the inductive method in the following sections.

\subsection{Structure Parameters of $N$-bar Goldberg Mechanisms}

Assume that the numbers of links and virtual loops of a Goldberg mechanism are $N$ and $L(L=N-3)$, respectively. $N$ is a natural number greater than four and the virtual loops refer to the loops of the original multi-loop Bennett mechanism. The lengths and stagger angles of the two side links, of the $j$-th $(j=1,2, \ldots, L)$ link connected to side link 1 , and of the frame are denoted by $a$ and $\alpha, b_{j}$ and $\beta_{j}$, and $d_{r}$ and $\beta_{r}$, respectively. The value ranges of $\alpha, \beta_{j}$ and $\beta_{r}$ are $\left(-90^{\circ}, 90^{\circ}\right)$, and $\alpha, \beta_{j}$ and $\beta_{r}$ take a positive value in the anticlockwise direction, and a negative value conversely. The structure parameters of an $N$-bar Goldberg mechanism can be expressed as

$$
\begin{gathered}
a_{i}=a_{N-1}, \quad \alpha_{i}=\alpha_{N-1}, \quad d_{r}=\sum_{j=1}^{L} b_{j}, \\
\beta_{r}=\sum_{j=1}^{L} \beta_{j},(j=1,2, \cdots, L), \\
\frac{\sin \alpha}{a}=-\frac{\sin \beta_{j}}{b_{j}}(j=1,2, \cdots, L), \\
\boldsymbol{\tau}_{a}=-\boldsymbol{\tau}_{b j},\left(\left|\boldsymbol{\tau}_{a}\right|=\left|\boldsymbol{\tau}_{b j}\right|=1\right)(j=1,2, \cdots, L) .
\end{gathered}
$$




\subsection{Displacement Equations of $N$-bar Goldberg}

\section{Mechanisms}

Assuming that $N$ is an odd number, $n=(N-1) / 2$ and $L=N-3$. Thus, the following displacement equations can be derived:

$$
\begin{gathered}
\sum_{i=1}^{n} \theta_{(2 i)}=(n-1) \pi, \\
\sum_{i=1}^{n-1} \theta_{(2 i+1)}-\theta_{1}-\theta_{N}=(n-2) \pi, \\
\tan \frac{\theta_{1}^{j}}{2} \tan \frac{\theta_{2}^{j}}{2}=\frac{\cos \left[\left(\beta_{j}-\alpha\right) / 2\right]}{\cos \left[\left(\beta_{j}+\alpha\right) / 2\right]}=k_{j} \quad(j=1,2 \ldots, L) .
\end{gathered}
$$

Assuming that $N$ is an even number, $n=N / 2$ and $L=N-3$. Thus, the following displacement equations can be derived:

$$
\begin{gathered}
\sum_{i=1}^{n-1} \theta_{(2 i)}-\theta_{N}=(n-2) \pi, \\
\sum_{i=1}^{n-1} \theta_{(2 i+1)}-\theta_{1}=(n-2) \pi, \\
\tan \frac{\theta_{1}^{j}}{2} \tan \frac{\theta_{2}^{j}}{2}=\frac{\cos \left[\left(\beta_{j}-\alpha\right) / 2\right]}{\cos \left[\left(\beta_{j}+\alpha\right) / 2\right]}=k_{j} \quad(j=1,2 \ldots, L) .
\end{gathered}
$$

Selecting side links 1 and $(N-1)$ as the input-link and output-link, respectively, $\theta_{1}=\theta_{1}^{\mathrm{I}}, \theta_{2}=\theta_{2}^{\mathrm{I}}, \theta_{N-1}=\theta_{3}^{L}$, and $\theta_{N}=\theta_{4}^{L}$. Then it can be derived that

$$
\begin{gathered}
\tan \frac{\theta_{1}}{2} \tan \frac{\theta_{N}}{2}=\frac{\cos \left[\left(\beta_{1}-\alpha\right) / 2\right]}{\cos \left[\left(\beta_{1}+\alpha\right) / 2\right]} \frac{\cos \left[\left(\beta_{2}-\alpha\right) / 2\right]}{\cos \left[\left(\beta_{2}+\alpha\right) / 2\right]} \ldots \\
\frac{\cos \left[\left(\beta_{L}-\alpha\right) / 2\right]}{\cos \left[\left(\beta_{L}+\alpha\right) / 2\right]}=k_{1} k_{2} \cdots k_{L},
\end{gathered},
$$

which is the input-output displacement equation expressed by the interior angles of side links 1 and $(N-1)$ of an $N$-bar Goldberg mechanism.

It can be known from the above analyses that there are two independent linear equations and $L \quad(L=N-3)$ independent nonlinear trigonometric equations for an $\mathrm{N}$-bar Goldberg mechanism, so the total number of independent equations of the mechanism is $n_{z}=L+2=N-3+2=N-1$. The loop rank of the $N$-bar Goldberg mechanism is also equal to $\mathrm{N}-1$, and Moroskine declared that the loop rank of a mechanism is equal to the number of closed-loop independent displacement equations of the mechanism, which shows that the definition of loop rank given by Moroskine is applicable to both general mechanisms and the mechanisms with the rank more than six, and it is the most universal one at present.

\section{Verification of the General Displacement Equations of $N$-bar Goldberg Mechanisms}

\subsection{Verification of the General Displacement Equations with an Odd $N$}

In this section, the generality of Eqs. (60) and (61) will be verified by assigning a series of odd values to $N$.

If $N=5$, there exist $n=(N-1) / 2=2$ and $L=5-3=2$, so it can be obtained from Eqs. (60) and (61) that $\theta_{2}+\theta_{4}=\pi$ and $\theta_{3}-\theta_{1}-\theta_{5}=0$.

If $N=7$, there exist $n=(N-1) / 2=3$ and $L=7-3=4$, so it can be obtained from Eqs. (60) and (61) that $\theta_{2}+\theta_{4}+$ $\theta_{6}=2 \pi$ and $\theta_{3}+\theta_{5}-\theta_{1}-\theta_{7}=\pi$.

If $N=9$, there exist $n=(N-1) / 2=4$ and $L=9-3=6$, so it can be obtained from Eqs. (60) and (61) that $\theta_{2}+\theta_{4}+\theta_{6}$ $+\theta_{8}=3 \pi$ and $\theta_{3}+\theta_{5}+\theta_{7}-\theta_{1}-\theta_{9}=2 \pi$.

If $N=11$, there exist $n=(N-1) / 2=5$ and $L=11-3=8$, so it can be obtained from Eqs. (60) and (61) that $\theta_{2}+\theta_{4}+\theta_{6}+\theta_{8}+\theta_{10}=4 \pi$ and $\theta_{3}+\theta_{5}+\theta_{7}+\theta_{9}-\theta_{1}-\theta_{11}=3 \pi$.

By analogy, an infinite number of Goldberg mechanisms with odd numbers of links can be obtained. The displacement equations of those Goldberg mechanisms obtained by Eqs. (60) and (61) are in accordance with those derived by analyzing the mechanisms, which verifies the generality and correctness of Eqs. (60) and (61).

\subsection{Verification of the General Displacement Equations with an Even $N$}

In this section, the generality of Eqs. (63) and (64) will be verified by assigning a series of even values to $N$.

If $N=4$, there exist $n=N / 2=2$ and $L=4-3=1$, so it can be obtained from Eqs. (63) and (64) that $\theta_{2}-\theta_{4}=0$ and $\theta_{3}-\theta_{1}=0$.

If $N=6$, there exist $n=N / 2=3$ and $L=6-3=3$, so it can be obtained from Eqs. (63) and (64) that $\theta_{2}+\theta_{4}-\theta_{6}=\pi$ and $\theta_{3}+\theta_{5}-\theta_{1}=\pi$.

If $N=8$, there exist $n=N / 2=4$ and $L=8-3=5$, so it can be obtained from Eqs. (63) and (64) that $\theta_{2}+\theta_{4}+\theta_{6}-$ $\theta_{8}=2 \pi$ and $\theta_{3}+\theta_{5}+\theta_{7}-\theta_{1}=2 \pi$.

If $N=10$, there exist $n=N / 2=5$ and $L=10-3=7$, so it can be obtained from Eqs. (63) and (64) that $\theta_{2}+\theta_{4}+$ $\theta_{6}+\theta_{8}-\theta_{10}=3 \pi$ and $\theta_{3}+\theta_{5}+\theta_{7}+\theta_{9}-\theta_{1}=3 \pi$.

By analogy, an infinite number of Goldberg mechanisms with even numbers of links can be obtained. The displacement equations of those Goldberg mechanisms obtained by Eqs. (63) and (64) are the same as those derived by analyzing the mechanisms, which verifies the generality and correctness of Eqs. (63) and (64).

It can be seen from the above analyses that the angular 
displacement equations of $N$-bar Goldberg mechanisms with odd and even links have generality. The tangent product equations of all the virtual loops of an $N$-bar Goldberg mechanism have the same form. For each additional link of an $N$-bar Goldberg mechanism, a virtual loop is added, which leads to an additional tangent product equation. For the sake of space, the correctness of the tangent product equations of an $\mathrm{N}$-bar Goldberg mechanism is not verified here.

\subsection{Consistency Test Between the Theoretical and} Measured Values of Angular Displacements

A left-handed nine-bar Goldberg mechanism is shown in Figure 12. A set of structure parameters are given as $a=120 \mathrm{~mm}, \quad \alpha=-20^{\circ}, \quad b_{1}=b_{2}=b_{3}=b_{4}=b_{5}=b_{6}=120 \mathrm{~mm}, \quad$ and $\beta_{1}=\beta_{2}=\beta_{3}=\beta_{4}=\beta_{5}=\beta_{6}=20^{\circ}$. Let the input parameter $\theta_{1}=92.15^{\circ}$, and it can be deduced from Eq. (56) that $\tan \frac{92.15^{\circ}}{2} \tan \frac{\theta_{9}}{2}=\left(\frac{\cos \left[\left(20^{\circ}-\left(-20^{\circ}\right)\right) / 2\right]}{\cos \left[\left(20^{\circ}+\left(-20^{\circ}\right)\right) / 2\right]}\right)^{6}$. Solving this equation yields $\theta_{9}=67.101^{\circ} \approx 67.10^{\circ}$, which is consistent with the measured value of $\theta_{9}$ shown in Figure 12.

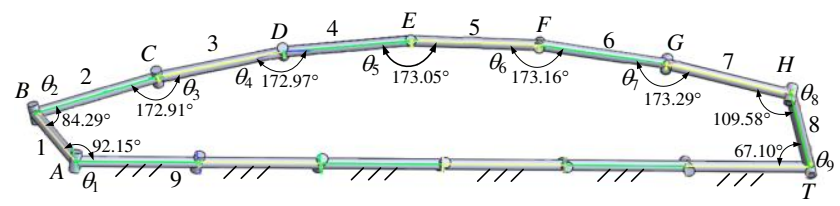

Figure 12 Displacement Parameters of Nine-bar Goldberg Mechanism with the Stagger Angle $\alpha=-20^{\circ}$

The measured values of $\theta_{2}, \theta_{4}, \theta_{6}$, and $\theta_{8}$ are $84.29^{\circ}$, $172.97^{\circ}, 173.16^{\circ}$, and $109.58^{\circ}$, respectively. Substituting $\theta_{2}=84.29^{\circ}, \theta_{4}=172.97^{\circ}, \theta_{6}=173.16^{\circ}$, and $\theta_{8}=109.58^{\circ}$ into Eq. (49) yields $\quad \theta_{2}+\theta_{4}+\theta_{6}+\theta_{8}=84.29^{\circ}+172.97^{\circ}+173.16^{\circ}$ $+109.58^{\circ}=540^{\circ}$ (i.e., $3 \pi$ ), so the measured values and theoretical values obtained by Eq. (49) of $\theta_{2}, \theta_{4}, \theta_{6}$, and $\theta_{8}$ are consistent.

The measured values of $\theta_{1}, \theta_{3}, \theta_{5}, \theta_{7}$, and $\theta_{9}$ are $92.15^{\circ}$, $172.91^{\circ}, 173.05^{\circ}, 173.29^{\circ}$, and $67.10^{\circ}$, respectively. Substituting $\theta_{1}=92.15^{\circ}, \theta_{3}=172.91^{\circ}, \theta_{5}=173.05^{\circ}, \theta_{7}=173.29^{\circ}$, and $\theta_{9}=67.10^{\circ}$ into Eq. (48) yields $\theta_{3}+\theta_{5}+\theta_{7}-\theta_{1}-\theta_{9}=$ $172.91^{\circ}+173.05^{\circ}+173.29^{\circ}-92.15^{\circ}-67.10^{\circ}=360^{\circ}$ (i.e., $2 \pi$ ), indicating that the measured values of $\theta_{1}, \theta_{3}, \theta_{5}, \theta_{7}$, and $\theta_{9}$ coincide with the theoretical values obtained by Eq. (48).

Substituting $\theta_{1}=92.15^{\circ}$ into Eq. (50) leads to $\tan \frac{92.15^{\circ}}{2} \tan \frac{\theta_{2}}{2}=\frac{\cos \left[\left(20^{\circ}-\left(-20^{\circ}\right)\right) / 2\right]}{\cos \left[\left(20^{\circ}+\left(-20^{\circ}\right)\right) / 2\right]}$. It can be solved from the above equation that $\theta_{2}=84.295^{\circ}$ with the error of $0.005^{\circ}$. Substituting $\theta_{1}=92.15^{\circ}$ and $\theta_{2}=84.29^{\circ}$ into
Eq. (51) yields $\tan \frac{180^{\circ}-84.29^{\circ}}{2} \tan \frac{\theta_{3}-92.15^{\circ}}{2}=$ $\frac{\cos \left[\left(20^{\circ}-\left(-20^{\circ}\right)\right) / 2\right]}{\cos \left[\left(20^{\circ}+\left(-20^{\circ}\right)\right) / 2\right]}$, which gives that $\theta_{3}=172.9069^{\circ} \approx$ $172.91^{\circ}$. Thus, the measured value of $\theta_{3}$ is consistent with its theoretical value solved by Eq. (51). Similarly, the correctness of the tangent product equations of other virtual loops can be verified in turn.

Therefore, the theoretical analysis and measurement of angular displacements have verified the correctness of the general displacement equations of $N$-bar Goldberg mechanisms.

\section{Conclusions}

In this study, the displacement equations of the eight-bar and nine-bar Goldberg mechanisms are constructed with interior angles of adjacent links, on the basis of the closed-loop displacement equations of Bennett mechanism. Furthermore, the general displacement equations of $N$-bar Goldberg mechanisms are deduced. The following conclusions can be drawn:

(1) An infinite number of single-loop $N$-bar $(N>4)$ Goldberg mechanisms with one DOF can be obtained by removing the common side links of adjacent loops from the multi-loop Bennett mechanisms in which all loop frames are collinear.

(2) For an $N$-bar $(N>4)$ Goldberg mechanism, the rank of loop, the numbers of displacement parameters and independent displacement equations are $(N-1), N$ and $(N-1)$, respectively. Thus, the definition given by Moroskine that "the loop rank of a mechanism is equal to the number of closed-loop independent displacement equations of the mechanism" is applicable to the mechanisms with $d \leqslant 6$ or $d>6$, which shows that Moroskine's definition is the most universal one among the various definitions of loop rank.

(3) For the $N$-bar $(N>8)$ Goldberg mechanisms, the loop rank and the number of independent displacement equations are larger than six, and the loop rank can be infinite. There exist an infinite number of single-DOF mechanisms with the rank more than six.

(4) The closed-loop displacement equations of $N$-bar Goldberg mechanisms proves that analyzing the trajectory circles of links is an effective method for judging whether the Goldberg mechanisms with the rank more than six have the constraints of Bennett mechanism.

(5) The consistency between the theoretical and measured displacement values of adjacent links of the nine-bar 
Goldberg mechanism proves the correctness and practicability of the displacement equations expressed by interior angles, as well as the correctness of the theoretical analyses in this paper.

\section{Declaration}

\section{Acknowledgements}

The authors sincerely thanks to Professor Yong-Sheng Zhao of Yanshan University for his critical discussion and reading during manuscript preparation.

\section{Funding}

Supported by the Natural Science Foundation of Hebei Province (Grant No. E2011203193).

\section{Availability of data and materials}

The datasets supporting the conclusions of this article are included within the article.

\section{Authors' contributions}

The author' contributions are as follows: Ling Lu was in charge of the whole trial; Yi-Tong Zhang was responsible for the main analysis; Wen-Lan Liu wrote the manuscript; Yan-Wen Li, Bo Hu, De-Jun Mu, and Wen-Juan Lu assisted with the analysis and discussion.

\section{Competing interests}

The authors declare no competing financial interests.

\section{Consent for publication}

Not applicable

\section{Ethics approval and consent to participate}

Not applicable

\section{References}

[1] Bennett G T. A new mechanism. Engineering, 1903, 76: 777-778

[2] Bennett G T. The parallel motion of Sarrut and some allied mechanisms. Philos Mag, 1905, 6(9): 803-810

[3] Bennett G T. Deformable octahedra. Proc Lond Math Soc (second series), 1911, 10(1): 309-343

[4] Bennett G T. The skew isogram mechanism. Proc London Math Soc, 1914, 23(1): 151-173

[5] Myard F E. Various titles. Acad Sci, Paris C R Heb des Seances, 1930, 1491: 190-192

[6] Myard F E. Contribution à la géométrie des systèmes articulés. Soc Math de Fr Bull, 1931, 59, 183-210

[7] Goldberg M. New five-bar and six-bar linkages in three dimensions. Trans ASME, 1943, 65: 649-663

[8] Baker J E. The Bennett, Goldberg and Myard linkages-in perspective. Mech Mach Theory, 1979, 14(4): 239-253
[9] Song C Y, Chen Y. A spatial 6R linkage derived from subtractive Goldberg 5R linkages. Mech Mach Theory, 2011, 46: 1097-1106

[10] Wohlhart K. Merging two general Goldberg 5R linkages to obtain a new 6R space mechanism. Mech Mach Theory, 1991, 26: 659-668

[11] Chen Y, You Z. Spatial 6R linkages based on the combination of two Goldberg 5R linkages. Mech Mach Theory, 2007, 42: 1484-1498

[12] Song C Y, Chen Y. Multiple linkage forms and bifurcation behaviours of the double-subtractive-Goldberg 6R linkage. Mech Mach Theory, 2012, 57: 95-110

[13] Gogu G. Mobility of mechanisms: a critical review. Mech Mach Theory, 2005, 40: 1068-1097

[14] Kong X W, Gosselin C M. Type synthesis of parallel mechanisms with multiple operation modes. ASME J Mech Design, 2007, 129(6): 595-601

[15] Gogu G. Structural synthesis of fully-isotropic translational parallel robots via theory of linear transformations. Eur J Mech A Solids, 2004 (23): 1021-1039

[16] Huang Z, Liu J F, Li Y W. On the degree of freedom-the general formula of the degree of freedom which has been searched for 150 years. Beijing: Science Press, 2011. (in Chinese)

[17] Huang Z, Fang Y F. Kinematic characteristics analysis of 3-DOF inparallel actuated pyramid mechanisms. Mech Mach Theory, 1996, 31(8): 1009-1018

[18] Yang T L, Liu A X, Luo Y F, et al, Theory and application of robot mechanism topology, Beijing: Science Press, 2012

[19] Gao F, Yang J L, Ge Q D. Type synthesis of parallel mechanisms based on GF set. Beijing: Science Press, 2011.

[20] Yang T L, Sun D J. A general DOF formula for parallel mechanisms and multi-loop spatial mechanisms. ASME J Mech Robot, 2012, 4 011001

[21] Zhang Y T, Mu D J. New concept and new theory of mobility calculation for multi-loop mechanisms. Sci China Tech Sci, 2010, 53: 1598-1604

[22] Zhang Y T, Li Y W, Wang L Y. A new formula of mechanism mobility based on virtual constraint loop. Sci China Tech Sci, 2011, 54: 2768 2775

[23] Zhang Y T, Lu W J, Mu D J, et al. Novel mobility formula for parallel mechanisms expressed with mobility of general link group. Chin J Mech Eng, 2013, 26(6): 1082-1090

[24] Deng Z, Huang H, Li B, et al. Synthesis of deployable/foldable single loop mechanisms with revolute joints. J Mech Robot, 2011, 3(3): 031006

[25] Liu X J, Wang J S. Parallel kinematics: types, kinematics and optimal design. Springer Tracts in Mechanical Engineering, Berlin Heidelberg: Springer-Verlag, 2014

[26] Moroskine Y F. General analysis of the theory of mechanisms. Moscow: Akad Nauk, SSSR, 1954

[27] Zhang Q X. Study on structural theory of spatial mechanisms. Chin J Mech Eng, 1961, 9(1): 7-32

[28] Zhao J S, Zhou K, Feng Z J. A theory of degrees of freedom for mechanisms. Mech Mach Theory, 2004, 39: 621-643

[29] Baker J E. Kinematic investigation of the deployable Bennett loop. ASME J Mech Des, 2007, 129: 602-610

[30] Song C Y, Chen Y, Chen I M. A 6R linkage reconfigurable between the line-symmetric Bricard linkage and the Bennett linkage. Mech Mach Theory, 2013, 70: 278-292

[31] Dalha C. Le Mécanisme de Bennett: propriétés et applications, Laboratoire de Mécanique appliquée, Besançon, France, 1982, 151151

[32] Yang F F, Chen Y, Kang R J, et al, Truss transformation method to 
obtain the non-overconstrained forms of 3D overconstrained linkages. Mech Mach Theory, 2016, 102: 149-166

[33] Hervé J M, Dahan M. The two kinds of Bennett's mechanisms. Proceedings of the Sixth World Congress on Theory of Machines and Mechanisms, New Delhi, India, 1983, 116-119

[34] Baker J E. Introducing the supplementary Bennett loop. P I Mech Eng C-J Mec, 2011, 225(2): 472-479

[35] Feng H J, Chen Y, Dai J S, et al. Kinematic study of the general planesymmetric Bricard linkage and its bifurcation variations. Mech Mach Theory, 2017, 116: 89-104

[36] Chen Y, Chai W H. Bifurcation of a special line and plane symmetric Bricard linkage. Mech Mach Theory, 2011, 46(4): 515-533

[37] Freudenstein F, Alizade R. On the degree-of-freedom of mechanisms with variable general constraint. Fourth World Congress on the Theory of Machines and Mechanisms, Sept. 8-12, 1975, Newcastle upon Tyne, UK

[38] Davies T H. Mechanical networks-I: Passivity and redundancy. Mech Mach Theory, 1983, 18: 95-101

\section{Biographical notes}

Yi-Tong Zhang, born in 1945, is currently a professor at School of Mechanical Engineering, Yanshan University, China. His research interest is the theory of mechanism structure.

E-mail: ytzhang@ysu.edu.cn

Ling Lu, born in 1964, is currently an associate professor at School of Mechanical Engineering, Yanshan University, China. She received her PhD degree from Yanshan University, China, in 2012. Her research interests include advanced manufacturing technology and robotics.

E-mail: luling@ysu.edu.cn
Wen-Lan Liu, born in 1990, is currently a teacher at Lanzhou University of Technology, China. She received her $\mathrm{PhD}$ degree from Yanshan University, China, in 2018. Her research interests include theory and application of parallel mechanisms.

E-mail: wenlanl@163.com

Yan-Wen Li, born in 1966, is currently a professor at School of Mechanical Engineering, Yanshan University, China. She received her $\mathrm{PhD}$ degree from Yanshan University, China. Her research interests include technology and application of robots.

E-mail: ywl@ysu.edu.cn

Bo Hu, born in 1982, is currently a professor at School of Mechanical Engineering, Yanshan University, China. He received his $\mathrm{PhD}$ degree from Yanshan University, China, in 2010. His research interests include theory of mechanism structure and robotics.

E-mail: hubo@ysu.edu.cn

De-Jun Mu, born in 1967, is currently an associate professor at School of Mechanical Engineering, Yanshan University, China. She received her $\mathrm{PhD}$ degree from Yanshan University, China. Her research interest is the theory of mechanism structure.

E-mail:djmu@ysu.edu.cn

Wen-Juan Lu, born in 1983, is currently an associate professor at School of Mechanical Engineering, Yanshan University, China. She received her $\mathrm{PhD}$ degree from Yanshan University, China, in 2015. Her research interest is the theory of mechanism structure. E-mail: luwenjuan@ysu.edu.cn 
Figures

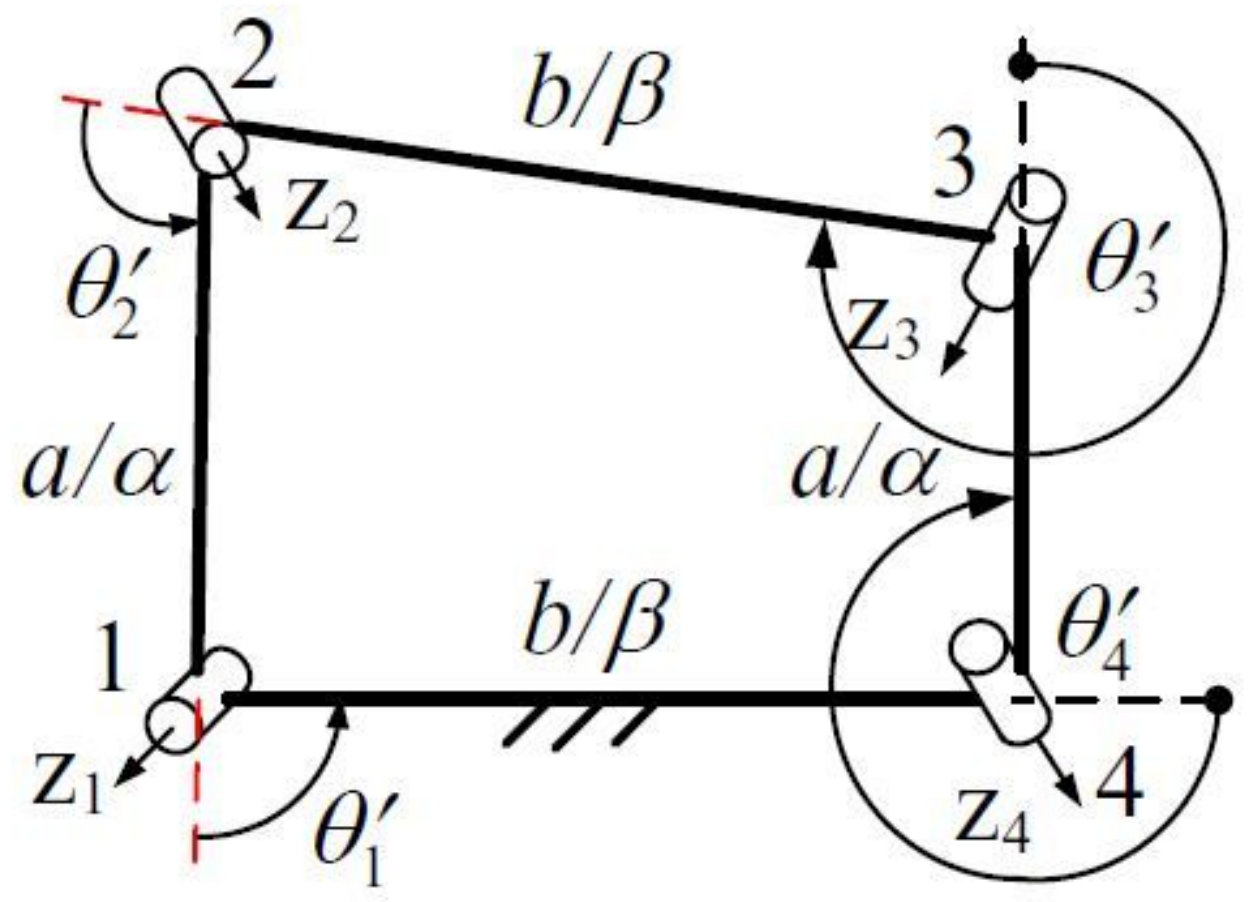

Figure 1

Bennett mechanism expressed by exterior angles

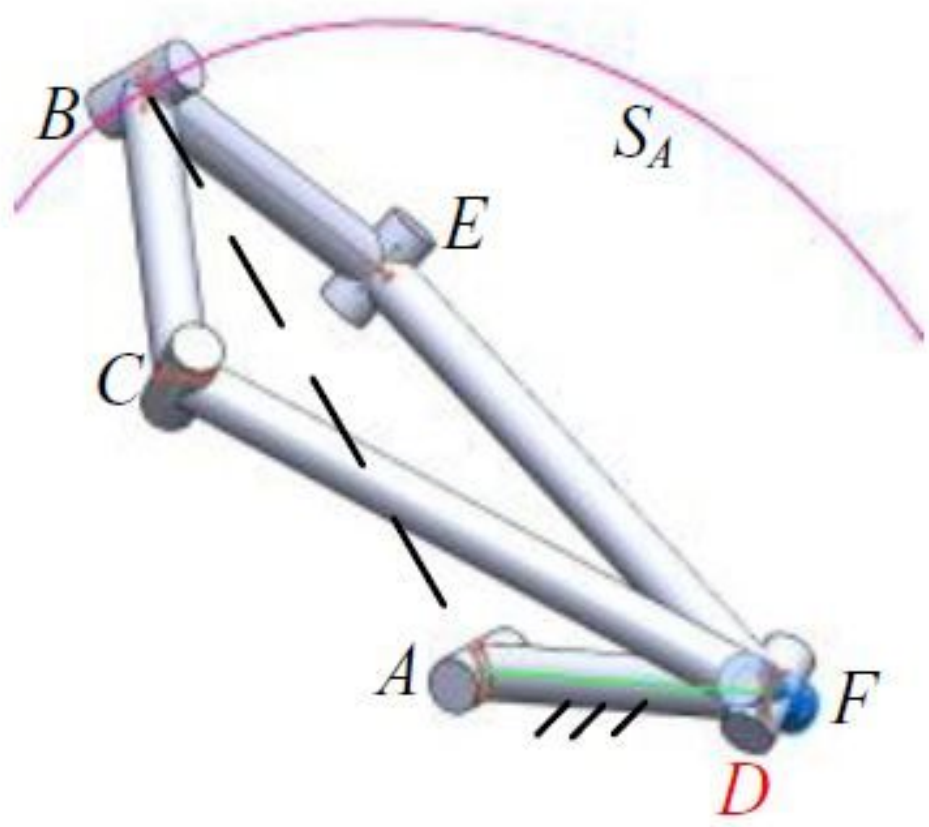


Figure 2

Myard mechanism

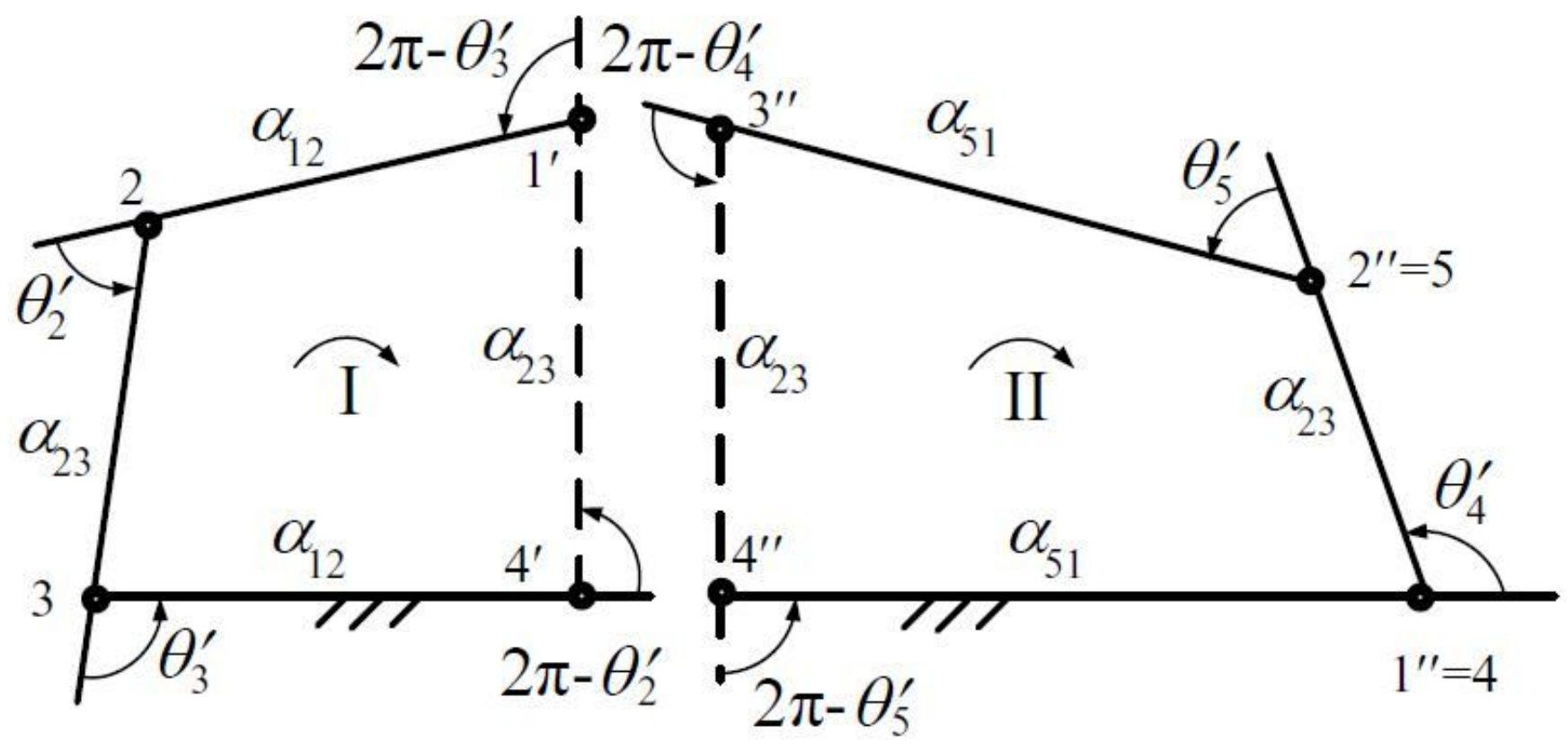

Figure 3

Five-bar Goldberg mechanism expressed by exterior angles

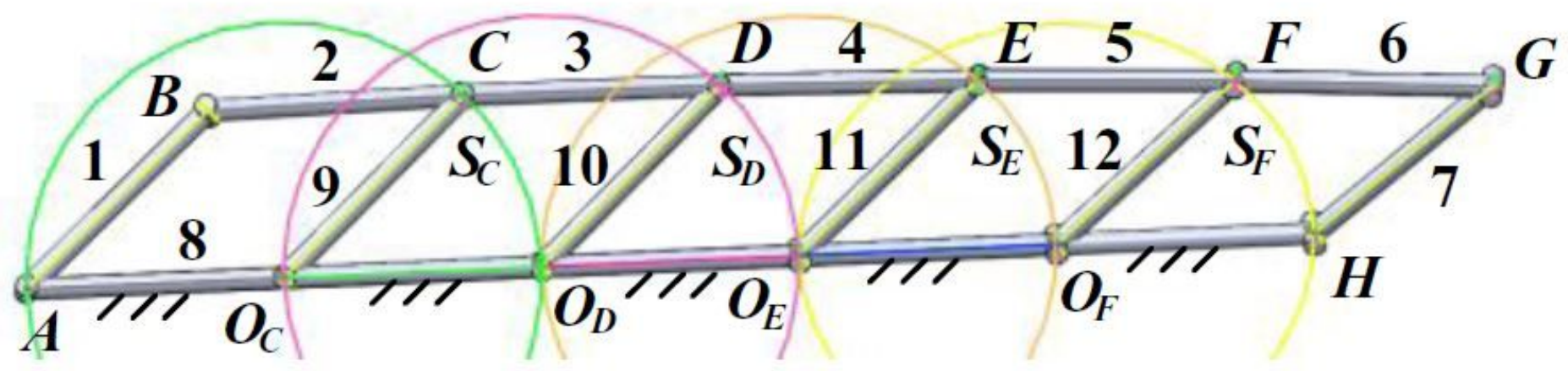

Figure 4

Five-loop Bennett mechanism 


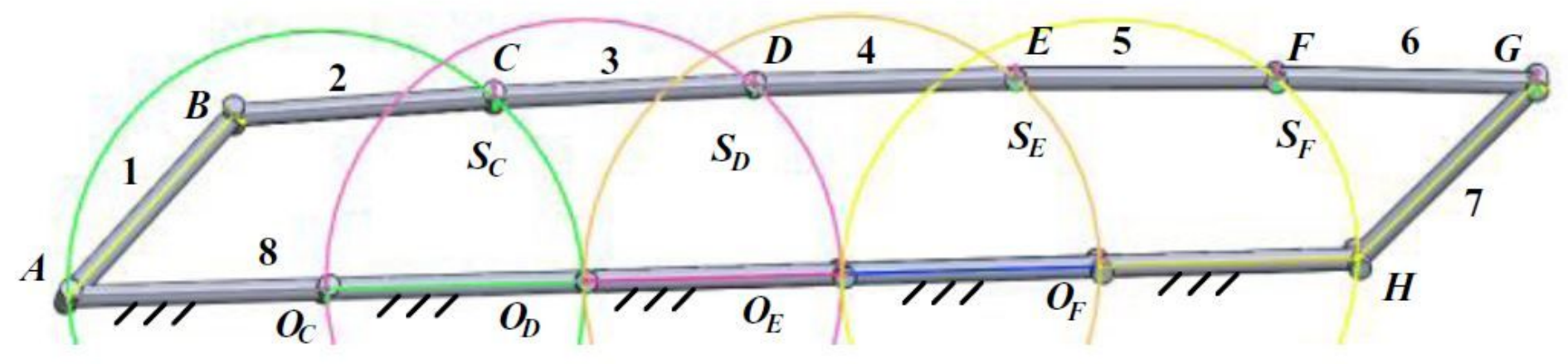

Figure 5

Eight-bar Goldberg mechanism

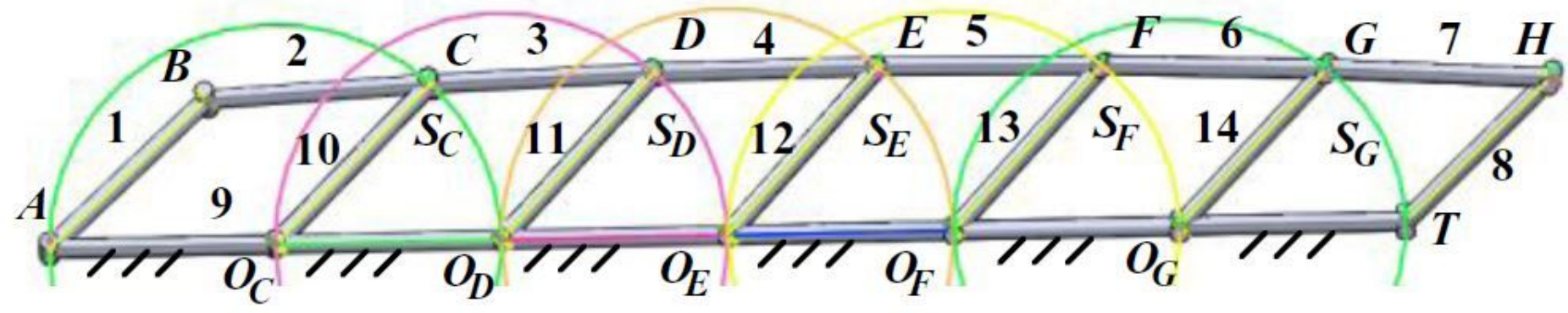

Figure 6

Six-loop Bennett mechanism

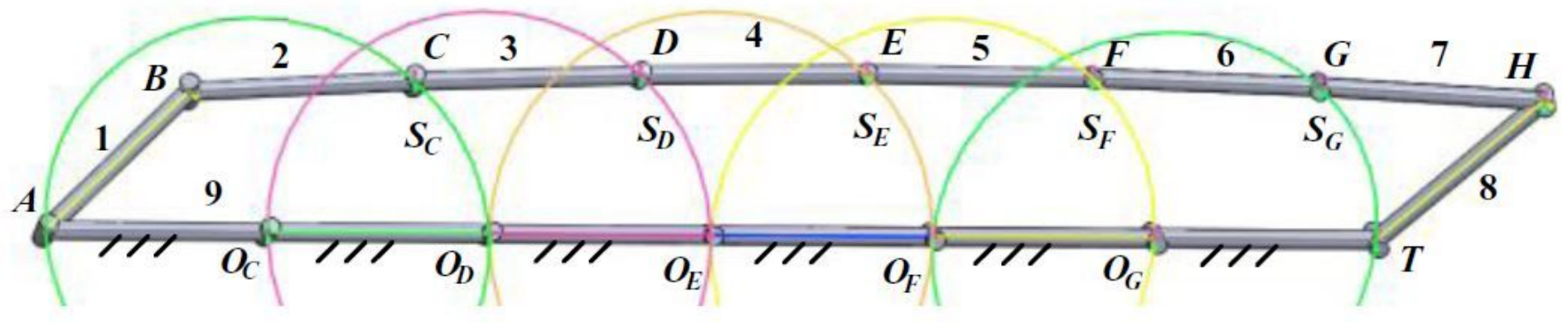

Figure 7

Nine-bar Goldberg mechanism 


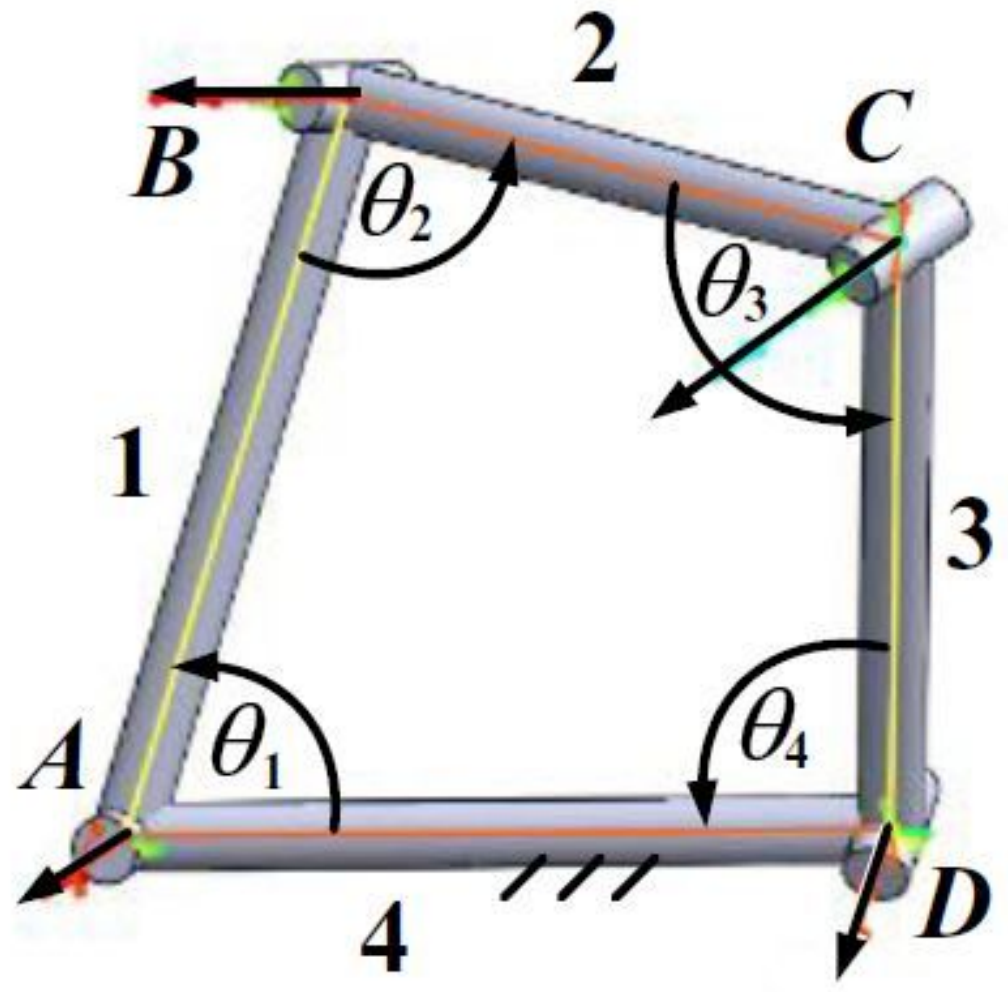

Figure 8

Bennett mechanism expressed by interior angles

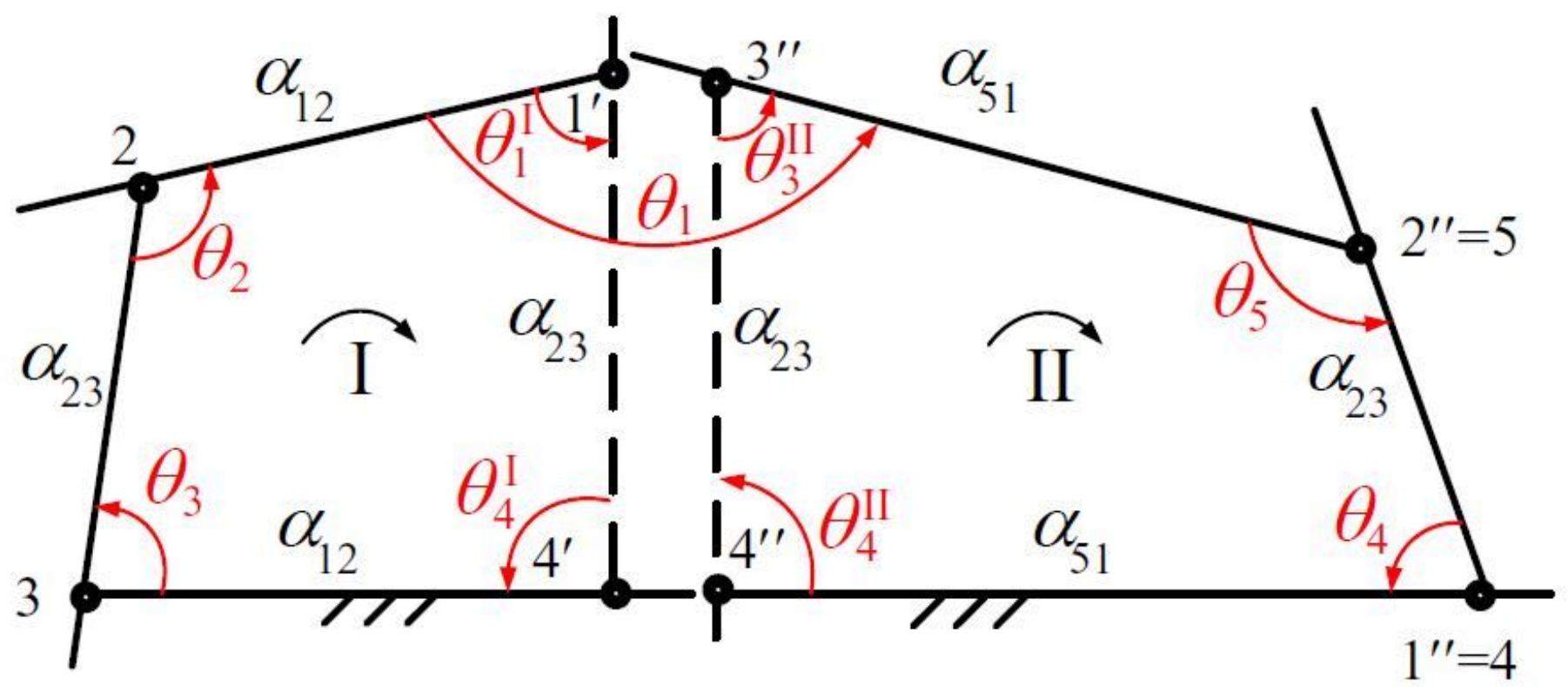

Figure 9

Five-bar Goldberg mechanism expressed by interior angles 


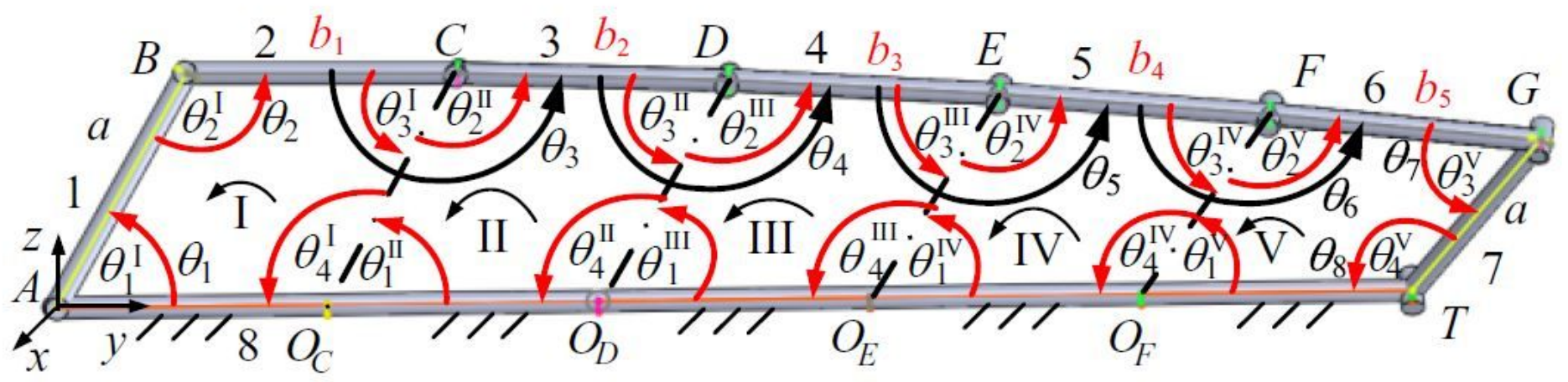

Figure 10

Displacement parameters of right-handed eight-bar Goldberg mechanism

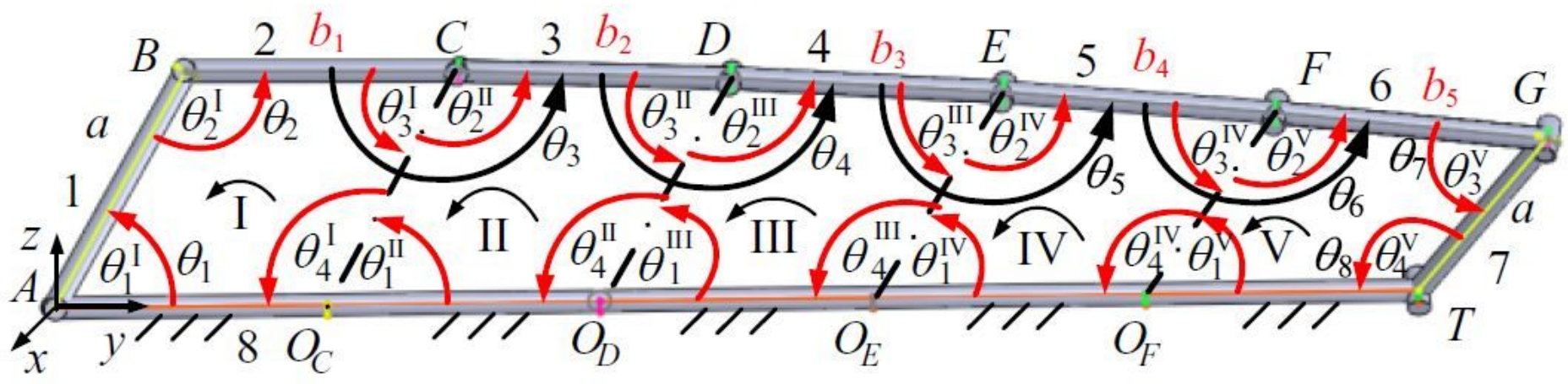

Figure 11

Displacement parameters of right-handed nine-bar Goldberg mechanism

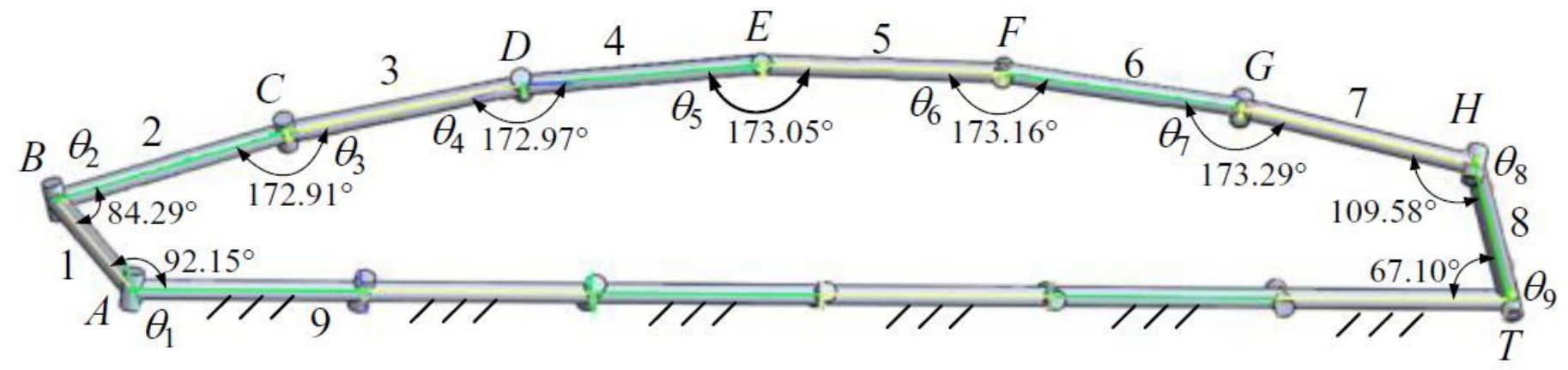

Figure 12

Displacement Parameters of Nine-bar Goldberg Mechanism with the Stagger Angle $\mathbb{\nabla}=-20^{\circ}$ 